\title{
EXPRESSION AND ORGANIZATION OF GEOGRAPHIC SPATIAL RELATIONS BASED ON TOPIC MAPS
}

\author{
H. J. Liang ${ }^{\text {a, }}$, H. Wang ${ }^{\text {a }}$, T. J. Cui ${ }^{\text {a, }}$, J. F. Guo ${ }^{\text {a }}$ \\ ${ }^{a}$ College of Urban and Environmental Science, Tianjin Normal University, Tianjin300387, China-Lianghj_nn@163.com
}

Commission IV, WG IV/3

KEY WORDS: Topic Maps, Spatial Relation, Topological Relation, Association, Topic Types, Occurrence

\begin{abstract}
:
Spatial Relation is one of the important components of Geographical Information Science and Spatial Database. There have been lots of researches on Spatial Relation and many different spatial relations have been proposed. The relationships among these spatial relations such as hierarchy and so on are complex and this brings some difficulties to the applications and teaching of these spatial relations. This paper summaries some common spatial relations, extracts the topic types, association types, resource types of these spatial relations using the technology of Topic Maps, and builds many different relationships among these spatial relations. Finally, this paper utilizes Java and Ontopia to build a topic map among these common spatial relations, forms a complex knowledge network of spatial relations, and realizes the effective management and retrieval of spatial relations.
\end{abstract}

\section{INTRODUCTION}

Tobler's First Law of Geography (TFL) thinks that: "everything is related to everything else, but near things are more related than distant things" (Tobler, 1970).In practice, in order to meet their own needs, different departments such as the government, the military, commercial enterprises describe and record the information of geographical objects and phenomena from different applications and perspectives, however it is very difficult to achieve the holographic expression of physical and humane geographical elements in a region. It is a hotspot in the field of geographic information research to establish the spatial relationships of different geographical data based on spatial relations, to effectively improve the ability to obtain valuable geographic information quickly, and to provide decision service for national economic construction. In recent years, people have made many rich achievements in the field of spatial relations. The descriptions of spatial relations are complex and diverse and there are various relationships among these description methods and models (e.g., there are some corresponding relations between four intersection model (4I), nine intersection model (9I) and CMB model of topological relation model). The ambiguity of direction concept, the hierarchical of direction relation reference system (Yan, 2002; Guo, 2007; Guo, 2014), and the localization, completeness and relativity of direction relations leads to the diversity of direction relation model and the complexity of direction relationship management. At present, the research on the spatial relations of topology, distance and direction is fragmented and it is difficult to establish connections among numerous spatial relations, which brings some difficulties for modeling, analyzing, querying of spatial data and teaching of GIS. Spatial relations are the core of complicated geographic networks and knowledge maps (Duan, 2013; Lu, 2014), in order to realize geographic knowledge maps, the first step is to build the spatial relation topic map. Topic Maps (TM) technology is a tool for building complex knowledge networks. Based on Topic Maps technology, this paper establishes a whole and united complex network of spatial relations which can integrate the knowledge of topology, direction and distance to realize the management and expression of spatial relations.

\section{TOPIC MAPS(TM)}

Topic Maps are the specification of organization, retrieval and navigation of semantic information on the Meta Layer $(\mathrm{Li}$, 2010). Known as the global positioning system in the information world (Liu, 2012), Topic Maps is an international standard being used to express and exchange knowledge and to link knowledge with information resources, it is mainly used in the field of information management and information exchange. Topic Maps not only can reveal the interrelations between information resources and knowledge concepts, but also can locate the knowledge concept. From the perspective of distinguishing information and knowledge, the structure of topic map can be divided into upper and lower layers, namely, information resource collection layer (lower layer) and knowledge map layer (upper layer). The structure diagram is shown in Figure 1.

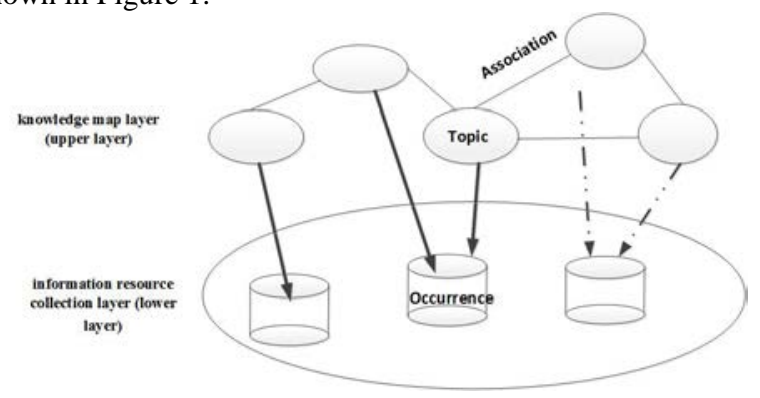

Figure 1. Two-layer structure schematic diagram of Topic Maps

Topic Maps contains three core elements: Topic, Association, and Occurrence (i.e., TAO). A topic is generally extracted after the analysis of different forms of resources according to the

\footnotetext{
${ }^{*}$ Corresponding author
} 
specific application fields and user requirements. An occurrence refers to an information resource associated with a topic at one or more levels. An association is a connection that can indicate a relationship among two or more topics. Topic Maps technology is widely used in tourism resources management, egovernment and knowledge management (Liu, 2006; Zhou, 2007; Eslami, 2013; Liu, 2012; Lv, 2007; Xia, 2010).

\section{SPATIAL RELATIONS AND TOPIC TYPES}

The construction of spatial relations topic maps involves creating spatial relation type topics, resource type topics, and association type topics. The first step is to establish the spatial relation types and their resource topics. Then we establish the association types on the basis of these types and topics to form a spatial relations topic map.

\subsection{Spatial relations}

Spatial relations include distance relations, direction relations and topological relations. The distance relations are used to describe the relative position among the spatial entities, reflecting the proximity of the space adjacent targets. In terms of the expression methods, the spatial distance can be divided into quantitative distance and qualitative distance. According to difference of the data structures used in GIS, the spatial distance can be also divided into the vector distance in Euclidean space and the grid distance in digital space. Since the definitions and understandings of distance vary from one application to another, people have proposed some extended spatial distances. The direction relations are used to express the order relationships of the spatial entities in space, and they need to be the descripted under a certain reference frame. The description model of direction relations is composed of reference object, primary object and reference frame. According to different applications, the reference frame can be divided into intrinsic reference frame, deictic reference frame and extrinsic reference frame (Retz-Schmidt, 1988). At present, the description models of direction relations include Minimum Enclosing Rectangle (MER), Minimum Bounding Rectangle (MBR), Triangular model, Double-crossed model (Freksa-Zimmermann), Conebased model, Projection-based model, Four Semi-Infinite Area (FSIA), 2-D string model, Direction relation matrix model , Voronoi-based model and so on. Topological relation analysis has been the most active research subject in spatial analysis. There are currently four-intersection Model (4I), nineintersection Model (9I) (Egenhofer, 1991), RCC-5, RCC-8], CBM, Dimensionally-Extended 9-Intersection Model (DE + 9IM)( Clementini,1995), RCC- 9 and CBM * model and so on.

\subsection{Topic types}

In the topic map, the topics can be divided into many groups according to different types, namely topic type. A topic can belong to more than one topic type, and a topic type in the topic map has also been identified as a topic. In the construction of the spatial relation topic map, the basic topic type of "spatial relation" is the superclass of the topic map, and the rest are the subclass of the class. The subtypes of "spatial relation" include topological relation, directional relation, and distance relation. Each subtype becomes a new topic type, and then determines its instance (i.e., the topic). The basic topic types of the spatial relation topic map include spatial relation (Spatial_Relation), topological relation (Topological_Relation), direction relation (Direction_Relation) and distance relation (Distance_Relation).
3.2.1 Topological relation topic types: The subtypes of the topological relation topic type include 4I (4_intersection), 9I (9_intersection), RCC5 (RCC5) and RCC8 (RCC8) (Wu, 2010).

(1) 4 I and 9I topic types

4I and 9I topic types include 6 kinds of subtypes: point-point topology (point_point_T), point-line topology (point_line_T), point-polygon topology (point_polygon_T), line-line topology (line_line_T), line-polygon topology (line_polygon_T) and polygon-polygon topology (polygon_polygon_T). Point-point topology topic type of 4I and 9I has 2 topics, namely overlap and disjoint. Point-line topology topic type of 4I and 9I has 3 topics, namely point_online, point_on_endpoint and disjoint. Point-polygon topology topic type of 4I and 9I has 3 topics, namely point_on_inner, points_on_boundary and disjoint. Polygon-polygon topology topic type of 4I and 9I has 8 topics, namley disjoint, contact, intersect, equal, cover, covered, contain and included_in; Line-line topology topic type of 4I has 16 topics, line-polygon topology topic type of $4 \mathrm{I}$ has 13 topics. Line-line topology topic type of 9I has 33 topics, line-polygon topology topic type of 9I has 19 topics. Topics of line-line topology topic type and line-polygon topology topic type of $4 \mathrm{I}$ and 9I are shown in Table 1 and Table 2.

\begin{tabular}{|c|c|c|}
\hline AI topics & It topics & Dessripticn \\
\hline Rllo & LL1 & Disjoint \\
\hline RII1 & L.21 and L.T.24 & Contact \\
\hline $\mathrm{R} 1 / 2$ & $\mathrm{~L}_{2}$ & Intersect \\
\hline RI13 & LL.22,LL.23 and LL.2S & The endpoints of $A$ coincide wih the endpoints of $B$, and $A$ and $B$ intersect \\
\hline Rl14 & LL8 and LL15 & The endpoints of $A$ are on $B$ \\
\hline Rlls & LL29 & $\begin{array}{l}\text { One endpoint of } A \text { is } \alpha_{1} B \text {, and another endpoint coincides with an endpoint of } \\
\text { B }\end{array}$ \\
\hline Rl16 & L.9,LL.10 and LL.16 & The endpoints of $A$ are $\operatorname{con} B$, and $A$ and $B$ intersect \\
\hline RI17 & L.30 and LL31 & $\begin{array}{l}\text { One encoint of } A \text { is on } B \text { and another endpoint concides with an endpoint of } \\
B \text {, and } A \text { and } B \text { intersect }\end{array}$ \\
\hline RII8 & $\mathrm{LL} 3$ and $\mathrm{LLA}$ & The endpoints of $B$ are on $A$ \\
\hline Rl19 & LL26 & $\begin{array}{l}\text { One conlpoint of } B \text { is } \infty \mathrm{A} \text {, and another endpoint coincides with an endpoint of } \\
\text { A }\end{array}$ \\
\hline Rll10 & $L S, L L 6$ and LL.7 & The endpoints of $B$ are on $A$, and $A$ and $B$ intersect \\
\hline Rll11 & LL27 and LL.28 & $\begin{array}{l}\text { One endpoint of } B \text { is on } A \text {,and ancher endpoint concides with an endpoint of } \\
A \text {, and } A \text { and } B \text { ireresect }\end{array}$ \\
\hline RII12 & $\begin{array}{l}\text { LL11, LL12, LL17 and } \\
\text { LL18 }\end{array}$ & $\begin{array}{l}\text { The endpoints of } B \text { are on A, and the endpoints of } A \text { are on B, and the } \\
\text { endpoints of } A \text { and } B \text { do not coincide }\end{array}$ \\
\hline Rl113 & L.32 & $\begin{array}{l}\text { One endpoint of } B \text { is on } A \text {, and one endpoint of } A \text { is } \propto \mathrm{B} \text {, and another } \\
\text { encipoint of } A \text { and } B \text { coincides, and } A \text { and } B \text { do not intersect }\end{array}$ \\
\hline Rl114 & $\begin{array}{l}\text { LL13,LL14,LL19 and } \\
\text { LL20 }\end{array}$ & $\begin{array}{l}\text { The cropoints of } B \text { are on } A \text {, and the endpoirts of } A \text { are on } B \text {, and } A \text { and } B \\
\text { intersect }\end{array}$ \\
\hline Rll15 & L.33 & $\begin{array}{l}\text { One endpoint of } B \text { is on } A \text {, and one endpoint of } A \text { is } \propto \text { an } B \text {, and another } \\
\text { encooint of } A \text { and } B \text { coincides, and } A \text { and } B \text { intersect }\end{array}$ \\
\hline
\end{tabular}

Table 1. Line-line Topological Relation of 4I and 9I

\begin{tabular}{|c|c|c|}
\hline I topics & 9I topics & Description \\
\hline Rlro & LR11 & Disjoint \\
\hline Rlr1 & LR12 and LR13 & The endpoints of $A$ are on the boundary of $B$ \\
\hline Rlr2 & LR42 & The endpoints of $A$ are on the boundary of $B$, and $A$ is in $B$ \\
\hline Rlr3 & LR44 & B contains $\mathrm{A}$ \\
\hline Rlr4 & LR46 & $B$ contains $A$, and one endpoint of $A$ is on the boundary of $B$ \\
\hline Rlrs & LR31 & $A$ part of $A$ is on the boundary of $B$, the other part is outside of $B$ \\
\hline Rlro & LR22,LR32 and LR33 & $A$ is on the boundary of $B$ \\
\hline RIr 7 & LR71 & $A$ intersects $B$, and $A$ has a portion on the boundary of $B$ \\
\hline Rlr8 & LR62, LR72 and LR73 & $\begin{array}{l}\mathrm{B} \text { intersects } \mathrm{A} \text {, and } \mathrm{A} \text { has a portion on the boundary of } \mathrm{B} \text {, and the } \\
\text { endpoints of } \mathrm{A} \text { are on the boundary of } \mathrm{B}\end{array}$ \\
\hline Rlr9 & LR64, LR74 and LR75 & $\begin{array}{l}B \text { intersects } A \text {, and } A \text { has a potion on the boundary of } B \text {, and the } \\
\text { endpoints of } A \text { are not on the boundary of } B\end{array}$ \\
\hline RIr10 & LR66 and LR76 & $\begin{array}{l}A \text { has a portion on the boundary of } B \text {, and one endpont of } A \text { is on the } \\
\text { boundary of } B \text {, and another endpoint is in } B\end{array}$ \\
\hline
\end{tabular}

Table 2. Line-polygon Topological Relation of 4I and 9I 
(2) RCC5 and RCC8 topic types

RCC5 topic type has a total of 5 topics, namely separated (DR), partial overlap (PO), true part (PP), equal (EQ) and anti-true part (PPI). RCC8 topic type has a total of 8 topics, namely not connected (DC), external connected (EC), partial overlap (PO), tangent true part (TPP), non-tangent true part (NTPP), equal (EQ), anti- tangent true part (TPPI) and anti- non-tangent true part (NTPPI).

3.2.2 Direction relation topic types: In this study, the topic type of direction relation includes three subtypes, namely Conebased (Cone_based), Double-crossed (Double_crossed) and MBR (MBR). Cone-based direction relation is classified as shown in Table 3.

\begin{tabular}{l|c|cc}
\hline 2-direction slice & \multicolumn{2}{|c|}{ 4-direction } & \multicolumn{2}{|c}{ 8-direction slice } \\
\hline & slice & East Northeast \\
Top and Bottom & West & West Northwest \\
Left and Right & South & South Southeast \\
& North & North Southwest \\
\hline
\end{tabular}

Table 3. Cone-based Direction Relation Classification

3.2.3 Distance relation topic types: The distance relation topic type includes 2 subtypes, namely the quantitative distance and the qualitative distance. The quantitative distance includes 2 subtypes, namely the vector distance and the grid distance, where the vector distance topic type includes a total of 6 topics : the distance from the point to point (point_ point _D), the distance from the point to line(point_ line _D), the distance from the point to polygon(point_ polygon _D), the distance from the line to line(line_ line _D), the distance from the line to polygon(line_polygon _D), and the distance from the polygon to polygon(polygon _ polygon_D). The grid distance topic type includes the board distance, the Manhattan distance, the octagonal distance and the slope. Qualitative distance includes a total of 3 topics: near, moderate and far.

\subsection{Association type}

To connect the relevant topics to form a knowledge network with a clear knowledge structure, it is necessary to bring together the topics through the interrelationships among them to help enhance the expression of the topic maps. According to the possible relationships among the various topic categories, four kinds of association types are obtained in the study: the parentchild association, the neighborhood association, the subset association and the corresponding association.

3.3.1 The parent-child association(Sub Relation):The topic type associats with subtypes at its next level in a parent-child hierarchy. For instance, spatial relation which is located in the top layer of the spatial relation topic map plays a role of father, while topological relation, direction relation and distance relation at the next level of spatial relation play the roles of the son, and the relationship between them is the parent-child association. The parent-child association reflects the hierarchy of spatial relations, which exists widely in spatial topological relations, direction relations and distance relations.
3.3.2 The neighborhood association: The neighborhood association is mainly manifested in the polygon-polygon topological relation and 8-direction slice relation, which is embodied by the conceptual neighborhood graph. Besides, there are neighborhood associations between the 8-direction slices of Cone-based direction relation model.

3.3.3 The Subset association: There is a set of subset relationships between the direction slices of Cone-based direction relation model. Specifically, the east, west, south and north of 4-direction slice are the subsets of right, left, bottom and top of 2-direction slice. The east, west, south and north of 8-direction slice are the subsets of east, west, south and north of 4-direction slice.

3.3.4 The corresponding association: There are spatial topological relation descriptions in line-line topological relations and line-polygon topological relations of $4 \mathrm{I}$ and 9I, and some of them are indistinguishable in the 4I, while they can be distinguished in the 9I. This relationship is embodied in the topic map with the corresponding association. The corresponding association between 4I and 9I is shown in Table 1 and Table 2. The corresponding association between RCC5 and RCC8 is shown in Table 4.

\begin{tabular}{llll}
\hline $\begin{array}{l}\text { RCC5 } \\
\text { topics }\end{array}$ & Description & $\begin{array}{l}\text { RCC8 } \\
\text { topics }\end{array}$ & Description \\
\hline DR & separated & $\begin{array}{l}\text { DC } \\
\text { EC }\end{array}$ & $\begin{array}{l}\text { not connected } \\
\text { external connected }\end{array}$ \\
\hline PO & partial overlap & PO & partial overlap \\
\hline PP & true part & TPP & $\begin{array}{l}\text { tangent true part } \\
\text { non-tangent true part }\end{array}$ \\
\hline EQ & equal & EQ & \multicolumn{1}{c}{ equal } \\
\hline PPI & anti-true part & TPPI & $\begin{array}{l}\text { anti-tangent true part } \\
\text { anti- non-tangent true } \\
\text { part }\end{array}$ \\
\hline
\end{tabular}

Table 4. RCC5 topic types and RCC8 topic types

\subsection{Occurrence}

Occurrence is also called the type of origin, which can be classified into pictures, digital resources and multimedia files according to the different ways of resource storage. In this study, occurrence is divided into two types: text description and picture. The" Description" in table 1--table 4 is the text description type of the topic, and the picture resource type of each spatial relation appears as a spatial relation diagram.

\section{VERIFICATION OF SPATIAL RELATION TOPIC} MAP

This paper uses the Java language and Ontopia to build a spatial relation topic map, and uses the Vizigator module features in Ontopia's OKSSample software to browse and query information. The topic map of spatial relation is verified as follows, where different associations are displayed using lines with different colors:

(1)Topic map of top spatial relation and Topic map of spatial topological relation show the parent-child association between 
superior and lower levels. As shown in Figure 2 and Figure 3, the parent-child association is represented by a brown line.

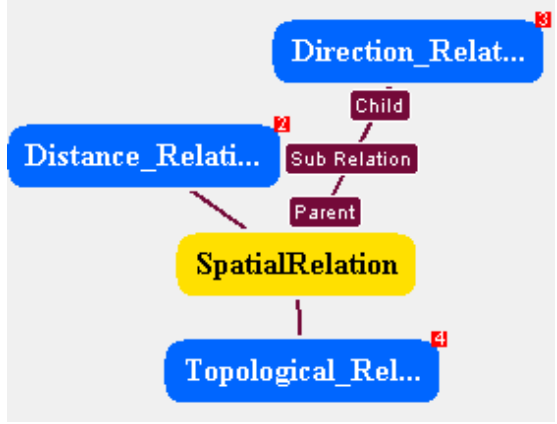

Figure 2. Topic map of top spatial relation

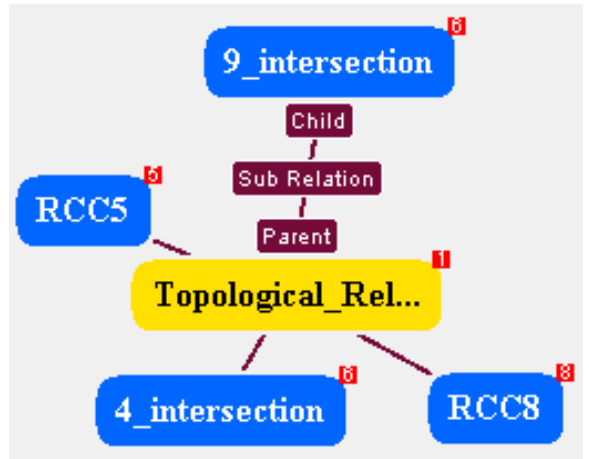

Figure 3. Topic map of spatial topological relation

(2) As shown in Figure 4, Topic map of polygon-polygon topological relations shows the neighborhood association between the polygon-polygon topological relations. These topics and associations constitute a conceptual neighborhood graph of the polygon-polygon topological relations. As shown in Figure 5, Topic map of neighborhood relations between direction tiles shows the neighborhood association between the 8-direction slices of Cone-based direction relation model. The neighborhood association is represented by a rose-red line.

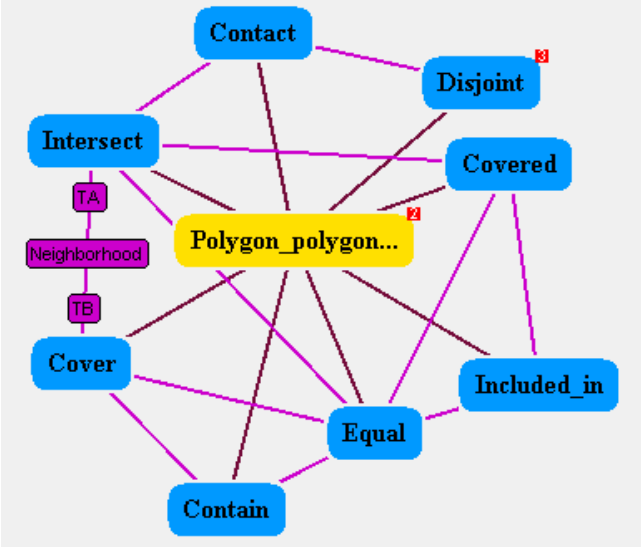

Figure 4. Topic map of polygon-polygon topological relations

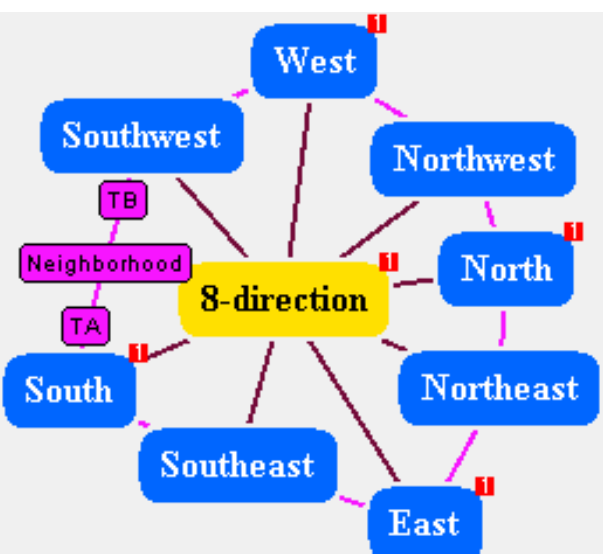

Figure 5. Topic map of neighborhood relations between direction tiles

(3) As shown in Figure 6, Topic map of Cone-based direction relation model depicts the subset associations between the direction relations. The subset association is represented by green lines.

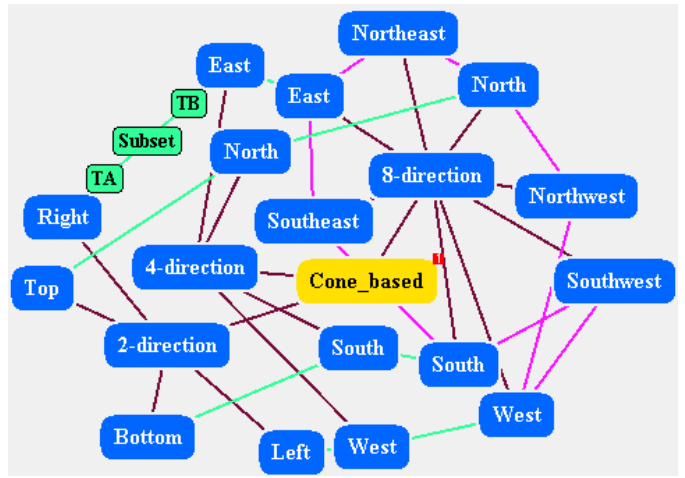

Figure 6. Topic map of Cone-based direction relation model

(4) As shown in Figure 7, Topic map of 4I model and 9I model depicts the corresponding associations between 4-intersection model and 9-intersection model. As shown in Figure 8, Topic map of RCC5 and RCC8 shows the corresponding association between RCC5 model and RCC8 model. The corresponding association is represented by red lines.

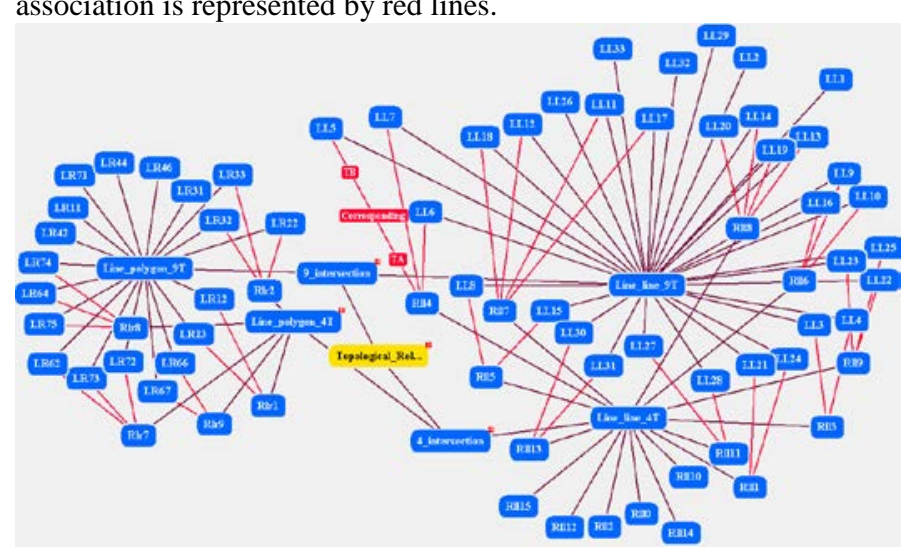

Figure 7. Topic map of 4I model and 9I model 


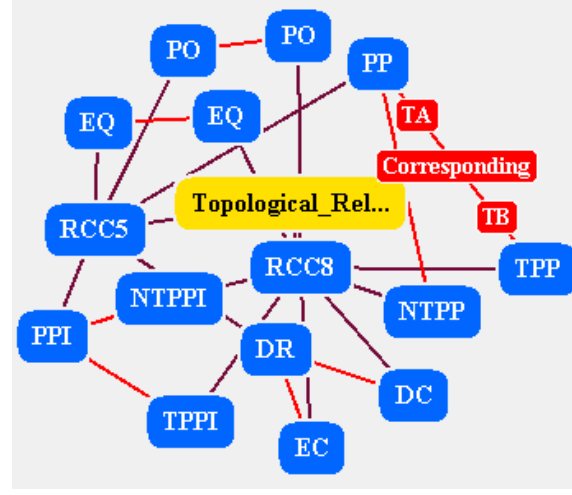

Figure 8. Topic map of RCC5 and RCC8

\section{INCONCLUSION}

This paper constructs a spatial relation knowledge network using Topic Maps technology, in which 155 topics were abstracted and 4 kinds of association types were established including the parent-child association, the neighborhood association, the corresponding association and the subset association. The topic map focuses on the construction of the topic network of the knowledge layer, covering the many categories of spatial relations, exposing the associations contained in the spatial relations objectively, forming a spatial relation topic map that integrates the topology, direction and distance relation. Because this is a complex network which realizes the integration management and expression of spatial relations, it has a profound significance in spatial relational reasoning and spatial knowledge expression. At the same time, it also provides a basis for the future study of the association and expression of massive geospatial knowledge

\section{ACKNOWLEDGEMENT}

We gratefully thank Prof. Cui for his valuable suggestions. This work was supported by the National Natural Science Foundation of China (41471314, 41101352).

\section{REFERENCES}

Clementini, E., Felice, P., 1995. A comparison of methods for representing topological relationships. Information SciencesApplications, 3(3), pp.149-178.

Duan, Y., Lu, F., 2013. Structural robustness of city road networks based on community. Computers. Environment and Urban Systems, 41(1), pp.75-87.

Egenhofer, M., Franzosa, R., 1991. Point-set topological spatial relations. International Journal of Geographical Information System, 5(2), pp. 161-174.

Eslami, S., Nazemi, E., 2013. An application of Topic Mapbased ontology generated from Wikipedia for query expansion. International Journal of Machine Learning and Computing, 3(4), pp. 357-360.

Guo, J., 2014. Multi-Scale Fuzzy Geospatial Analysis. Surveying and Mapping Press, Beijing, pp.94-95.

Guo, Q., Zheng, C., 2007. Improvement of cone-shaped spatial direction-relation model. Geomatics and Information Science of
Wuhan University, 32(1), pp.81-84.

Li, Y., Lian, X., Zhu, L., et al, 2010. A survey of topic maps and its application study (part 1/2). Digital Library Forum, 2010(5), pp.20-27.

Liu, D., 2012. Review of domestic research and application of Topic Maps. Library and Information Service, 56(5), pp. 62-65.

Liu, H., Wang, S., 2006. Topic Maps and discipline knowledge management. Computer Era, 2006(3), pp. 25-29.

Liu, X., Yang, Y., Yan, H., 2012. Research on the application of Topic Maps in public security information resources integration. Computer Applications and Software, 29(4), pp.206-208.

Lu, F., Zhang, H., 2014. Big data and generalized GIS. Geomatics and Information Science of Wuhan University, 39(6), pp.646-654.

Lv, Y., Wang, X., Tan, B., 2007.The research of e - government information resources organization based on Topic Maps. Journal of Library Science In China, 2007(4), pp. 73-76.

Retz-Schmidt, G., 1988.Various Views on Spatial Prepositions.AI Magazine, 9(2), pp. 95-105.

Tobler, W., 1970. A computer movie simulating urban growth in the Detroit region. Economic Geography, 46(2), pp.234-240.

Wu, C., Lv, G., 2010.Spatial topological relationships: an overview and analysis. Journal of Geo-Information Science, 2010 (8), pp.524-531.

Xia, L., Xu, C., 2010. The research of e - government gateway knowledge navigation system based on Topic Maps. Library Tribune, 2010(6), pp. 184-187.

Yan, H., Guo, R., 2002. A formal description research of directional relationships based on voronoi diagram. Developments In Surveying and Mapping, 27(1), pp.24-27.

Zhou, T., Zhou, Z., Huang, G., 2007. Research on Topic Mapbased domain knowledge management. Computer Engineering and Applications, 43(18), pp.225- 228. 